\title{
Nickel nanoparticles inside carbon nanostructures: atomistic simulation
}

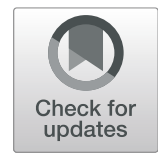

Liliya R. Safina ${ }^{1,2}$, Julia A. Baimova ${ }^{1,2^{*}}$ (i) and Radik R. Mulyukov ${ }^{1,2}$

\begin{abstract}
Ni nanoparticle on a graphene substrate, inside the fullerene and carbon nanotube was studied by molecular dynamics simulation technique. Morse interatomic potential have been used for $\mathrm{Ni}-\mathrm{Ni}$ and $\mathrm{Ni}-\mathrm{C}$ interactions, and AIREBO potential has been used for C-C interaction. The pairwise Morse potential was chosen for the description of the $\mathrm{Ni}-\mathrm{C}$ interaction because of its simplicity. It is shown that Morse potential can satisfactory reproduce the properties of graphene-nickel system. The effect of boundary conditions on the interaction of Ni nanoparticle and graphene sheet are investigated. It is shown, that if the edges of graphene plane are set to be free, coverage of Ni nanoparticle by graphene or just crumpling of graphene is observed depending on the size of nanoparticle. It is found, that Ni nanoparticle tend to attach to the carbon surface - graphene plane or the shell of fullerene and nanotube. Moreover, Ni nanoparticle induce the deformation of the surface of carbon polymorph. The obtained results are potentially important for understanding of the fabrication of metal-carbon composites and interaction between graphene and metal nanoparticles in such a system.
\end{abstract}

Keywords: Ni nanoparticle, Graphene, Molecular dynamics, Carbon nanostructures

\section{Introduction}

After graphene (Novoselov 2004; Geim and Novoselov 2007; Neto et al. 2009) and carbon nanotubes (Iijima and Ichihashi 1993) were first reported, they are considered to be an ideal material for future high-performance electronic devise applications, transistors, solar cells and many other applications. Currently, nanomaterials such as graphene and carbon nanotubes, have become more and more popular as reinforcing materials for metal composites (Neubauer et al. 2010). These graphene-metal hybrid structures are especially interesting because can display not only the individual properties of graphene but qualitatively new properties. Recently, nickel nanomagnets created in single-walled carbon nanotubes were obtained (Shiozawa et al. 2015). Among other metals, nickel (especially nanocrystalline) plays an important role in fabrication of the composites being used in many industrial applications, for example, for aerospace and chemical industry, in nanoelectronics, chemical cells, to name a few. One of the important advantages is that nickel exhibits

*Correspondence: julia.a.baimova@gmail.com

${ }^{1}$ Institute for Metals Superplasticity Problems of RAS, 39, Khalturina Str., 450001 Ufa, Russia

${ }^{2}$ Bashkir State University, 32, Validy Str., 450076, Ufa, Russia outstanding wettability for graphite powders and does not form an equilibrium carbide phase (Ip et al. 1998). From this point of view, combination of the advantages of graphene and $\mathrm{Ni}$ in the graphene-Ni composites can lead to the achievement of the better properties.

Graphene-Ni composites can be fabricated by laser sintering (Hu et al. 2016), in-situ growing using a powder metallurgy method (Jiang et al. 2018), jet electrodeposition (Ji et al. 2018), etc. Such composites have enhanced mechanical properties and can be used for superior mechanical performance (Hu et al.2016; Jiang et al. 2018); expected to have excellent radiation tolerance (Huang et al. 2018); can be used for hydrogen storage (Zhou et al. 2016; Gaboardi et al. 2014). Among the various graphene-Ni composites, special attention is paid to $\mathrm{Ni}$ nanoparticles (NP)-based graphene composites (Neiva et al. 2015; Mahale et al. 2014; Fu et al. 2013). A wide range of attention and applications can be explained by nickel NPs catalytic performance and its electronic properties. Moreover, presence of Ni NPs can activate the formation of new structural configurations like nanoscrolls (Bejagam et al. 2018; Savin et al. 2016). In spite of intensive research and a huge amount of collected experimental information, 
the investigation of interaction between metallic nanoparticles and carbon nanostructures is of high interest. Such studies can shed the light on the growth mechanism of carbon polymorphs on nanoparticles, on the process of catalysis and, in common, on the possibility of using such hybrid nanostructures for various applications, especially for the fabrication of graphene-metal composites.

The interest in modeling finite temperature, equilibrium, dynamic, and reactive properties of large systems at the atomic scale with sufficient accuracy has increased considerably in the last decades. One of the effective methods for analysis of properties and structure of as well carbon and metallic materials is molecular dynamics simulation. The (semi-)empirical approaches include Stillinger- and Weber-type models (Stillinger and Weber 1985; Marks 2000), empirical bond order potentials (Brenner et al. 2002; Los et al. 2005; van Duin et al. 2001; Tersoff 1986), and higher-order, so-called analytical bond order potentials (Pettifor and Oleinik 2000; Murdick et al. 2006; Gibson et al. 2010; Stuart et al. 2000), were employed for simulations. All of these potentials were successfully used for the investigation of various carbon and hydrocarbon systems (Baimova et al. 2017; Lisovenko et al. 2017; Krylova et al. 2016; Bai et al. 2017; Pedrielli et al. 2017; Zhan et al. 2016), while the determination of a potential for the $\mathrm{Ni}-\mathrm{C}$ interaction is a challenging and nontrivial task.

Molecular dynamics study of formation of metallofullerenes (Yamaguchi and Maruyama 1999) and singlewalled carbon nanotubes during catalysis in the presence of metal nanoparticle (Shibuta and Maruyama 2007) were conducted which shed the light on the growth of carbon nanostructures. In Verkhovtsev et al. (2014) ab-initio and bond-order many-body potential optimization of small Ni-hydrocarbon systems was applied for simulating the catalyzed growth of single-walled carbon nanotubes on nickel clusters. Application of the complex potential function for $\mathrm{Ni}-\mathrm{C}$ interaction definitely will lead to the quantitatively correct results, but the simulation of a big system with thousands of atoms require a lot of computation resources. Thus, in some approaches, simple pair interatomic potential, like Morse or Lennard-Jones, can be used (Yan et al. 2017; Katin et al. 2018; Galashev et al. 2019). As it was shown, Morse potential with the carefully chosen parameters can be used for the simulation of $\mathrm{C}-\mathrm{Ni}$ system and the results are in qualitative agreement with the results obtained by more complex methods, which was described in Katin et al. (2018); Galashev et al. (2019). Usage of the pairwise Morse potential will allow for the study of the atomistic details of the behaviour of carbon-Ni system, keeping a clear physical picture of the processes occurring in the system.

In the present work, the following issues are considered: (i) the possibility of application of Morse potential function with the parameters given in Katin et al. (2018) for investigation of carbon-Ni system behaviour; (ii) melting of the Ni NP at given Morse parameters and NP size; (iii) interaction between nickel NPs of different size with the three types of carbon nanopolymorphs; (iv) possibility of investigation of Ni NPs inside the pores of crumpled graphene. As a result, Ni nanoparticle inside carbon nanotube and fullerene and Ni nanoparticle on the graphene surface are studied by molecular dynamics simulation with the Morse potential function. Various boundary conditions are considered for Ni NP on graphene.

\section{Methods}

\section{Potential function}

The potential of the C-Ni system can be defines as the sum of three potential energies of carbon-carbon, carbonnickel and nickel-nickel interactions correspondingly:

$$
U_{\text {system }}=U_{C-C}+U_{C-N i}+U_{N i-N i}
$$

The first term is calculated using AIREBO potential (Stuart et al. 2000):

$$
U_{C-C}=\frac{1}{2} \sum_{i} \sum_{i \neq j}\left[U_{i j}^{R E B O}+U_{i j}^{L J}+\sum_{k \neq i, j} \sum_{l \neq i, j, k} U_{k i j l}^{T O R S I O N}\right],
$$

where $U_{i j}^{R E B O}$ is the hydrocarbon REBO potential developed in Brenner et al. (2002), $U_{i j}^{L J}$ term adds longer-ranged interactions using a form similar to the standard Lennard Jones potential, and $U_{k i j l}^{T O R S I O N}$ describes various dihedral angle preferences in hydrocarbon configurations.

Interatomic AIREBO potential has been widely employed in the molecular dynamics simulation of mechanical behaviour of graphene and graphene nanoribbon (Yang et al. 2018; Baimova JA et al. 2014), thermal conductivity of hybrid graphene (Liu et al. 2012), discrete breathers in diamond (Murzaev et al. 2017) etc.

The second and third terms of Eq. (1) are calculated using Morse potential

$$
U^{N i-C}(r)=D_{e}\left[\left(1-e^{-\beta\left(r-R_{e}\right)}\right)^{2}-1\right],
$$

where $D_{e}$ is the binding energy, $R_{e}$ - distance for potential energy minimum and $\beta$ - potential parameter. Morse potential was previously successfully used for the investigation of discrete breathers in two- and three-dimensional crystals (Dmitriev et al. 2010; Chetverikov et al. 2017), studying of the martensitic transformation (Dmitriev et al. 2017; Babicheva et al. 2015), hydrogen impurities in Pd and Ni (Poletaev et al. 2017), cutting process of Nibased superalloy using $\mathrm{SiC}$ tools (Hao et al. 2019) to name a few. Numerous molecular dynamics simulations 
of Ni nanoparticles are conducted using embedded atom method parametrization for interatomic potential that reproduces reasonably well the properties of $\mathrm{Ni}$ clusters. However, it should be noted, that such studies were devoted to single metal nanoparticles interacting with each other or different metal NP to study the interaction kinetics, melting process, to name a few (Henz et al. 2009; Sharma et al. 2018; Yousefia and Khoie 2015; Joshi et al. 2010). Even though application of Morse potential is the simplification of the real systems, it can provide results of physical relevance. Previously, simple pair interatomic potential was successfully used for the investigation of structural stability and energetics of nickel clusters, $\mathrm{Ni}_{n}$ ( $n=3-459)$; melting and fragmentation behaviors of $\mathrm{Ni}_{429}$ cluster where roles of the surface and core atoms in the melting and fragmentation process is discussed (Erkoc et al. 2000; Gunes and Erkoc 2000).

Morse parameters used in the present work are presented in Table 1. Mose parameters, developed in Girifalco and Weizer (687) are commonly used for the investigation of bulk Ni samples and should be carefully used for the studying of nano objects. However, in the present work Ni nanoparticles studied mostly in the interaction with the carbon nanopolymorphs and Morse parameters of $\mathrm{Ni}-\mathrm{C}$ interaction became more important from this point of view. Nevertheless, behaviour of the Ni nanoparticles also very important and should qualitatively satisfy the behaviour of real system. Thus, the whole structure is also studied with the help of ReaxFF (Reactive Force Field) potential based on highly accurate and benchmarking density functional studies developed recently for $\mathrm{Ni}-\mathrm{C}$ interaction (Bejagam et al. 2018). It should be mentioned, that to additionally check if Morse potential with the chosen parameters can satisfactory reproduce properties of $\mathrm{Ni}$, embedded-atom-method function for $\mathrm{Ni}$ (Foiles et al. 1986) is used to check the melting temperature of Ni NPs.

\section{Initial structures}

In Fig. 1a it is shown how the Ni NP is obtained from bulk crystal of face centered cubic Ni sample by simple cutting of the sphere with the radius $R$. Thus, at first, bulk Ni ample is created using LAMMPS, and then NP is cut using home-made programme code. Three NPs are obtained form the same bulk $f c c \mathrm{Ni}(111)$ sample to check the suitable NP size: $\mathrm{Ni}_{34}$ with the average diameter $\varnothing 7.5$ $\AA, \mathrm{Ni}_{382}(\varnothing 20 \AA)$ and $\mathrm{Ni}_{1192}(\varnothing 27 \AA)$. Similar approach was

Table 1 Morse parameters

\begin{tabular}{lllll}
\hline & $D_{e}, \mathrm{eV}$ & $R_{e}, \AA$ & $\beta, 1 / \AA$ & Ref. \\
\hline $\mathrm{Ni}-\mathrm{Ni}$ & 0.4205 & 2.78 & 1.4199 & (Girifalco and Weizer 687) \\
$\mathrm{C}-\mathrm{Ni}$ & 0.433 & 2.316 & 3.244 & (Katin et al. 2018; Galashev et al. 2019)
\end{tabular}

previously used in Erkoc et al. (2000); Gunes and Erkoc (2000). In the present work, only spherical nanoparticles are studied and the main attention is paid to the size of the NPs as the critical factor. As it was shown previously, NP curvature (Antoniammal and Arivuoli 2012) and chosen contact surface (Ryu et al. 2010) when interaction with the other structure is considered are of high importance. However, in the systems considered here, NPs are set close to carbon surface not directly on it and during simulation NP transformation took place and the surface reconstructs such as to form the ideal contact surface.

Periodic boundary conditions (PBC) are applied in all directions, but NP is placed in the center of large simulation cell to avoid the interaction between NPs in the neighbouring cells (see Fig. 1b). All the nanoparticles are relaxed at room temperature to reach the minimum potential energy. Relaxation leads to immediate change of the initial interatomic distance $2.49 \AA$ of bulk Ni to $\sim 2.7 \AA$ for NP. Analysis of the structural state sows that $\mathrm{Ni}_{34}$ NP, which initially have the same structural order as bulk $\mathrm{Ni}(111)$ sample, after relaxation became to be an unordered cluster of $\mathrm{Ni}$ atoms but still solid-like (see Fig. 1c). Two bigger NPs keep crystalline order of core atoms during relaxation. Potential energy of the surface atoms are much higher than of core atoms and for $\mathrm{Ni}_{34}$ nanocluster most of the atoms belong to the surface.

Three Ni-carbon systems are considered: Ni nanoparticle inside the fullerene, inside the carbon nanotube (CNT) and on graphene plane as shown in Fig. 2. Fullerenes of two different sizes are considered $-C_{240}$ and $C_{540}$. To study the movement of the NP inside fullerene, $\mathrm{Ni}_{34}$ cluster is chosen with the diameter $d_{N P}$ close to diameter of $\mathrm{C}_{240}$ and two times smaller than diameter of $\mathrm{C}_{540}$ as it can be seen from Fig. 2a. The same is for CNTs: diameter of CNT $(11,11)$ close to $d_{N P_{34}}$ and diameter of CNT $(21,21)$ is two times bigger (see Fig. 2b). It should be mentioned that $d_{C N T_{(11,11)}}=d_{C_{240}}$. Boundary condition for NP-fullerene system is the same as for single NP and size of the simulation cell along $x, y$ and $z 5$ times bigger than $d_{F}$ to avoid the interaction (see Fig. 1c - fullerene instead of NP). Boundary conditions for NP-CNT system are periodic along $x$ and $y$-axis with the size of the simulation cell 5 times bigger than $d_{C N T}$ while along $z$-axis usual PBC are applied (see Fig. 2b).

In Fig. 2c, initial structures of Ni nanoparticle $\mathrm{Ni}_{382}$ on graphene plane are shown. The size of graphene sheet is $60.3 \times 63 \AA$, and all three nanoparticles are considered $\mathrm{Ni}_{34}, \mathrm{Ni}_{382}$ and $\mathrm{Ni}_{1192}$. For graphene layer two types of periodic conditions were used: $\mathrm{PBC}$ and free edges. Under $\mathrm{PBC}$, edges of graphene cannot move which means that infinite graphene plane is considered. At free edges, graphene plane can bend and crumple around the particle which allow to study the coverage of the NP by graphene. The size of graphene sheet chosen close to the radius of 
a

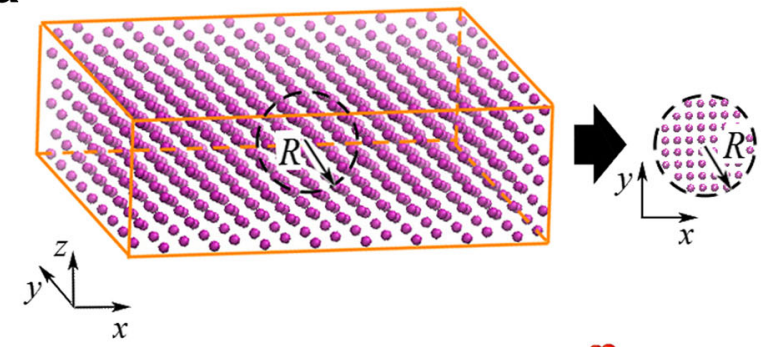

C

$\mathrm{Ni}_{1192}$
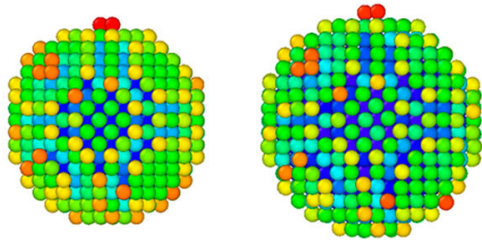

$\mathrm{Ni}_{382}$

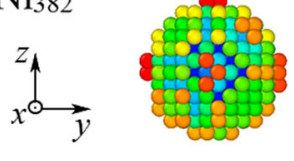

$\mathrm{Ni}_{34}$
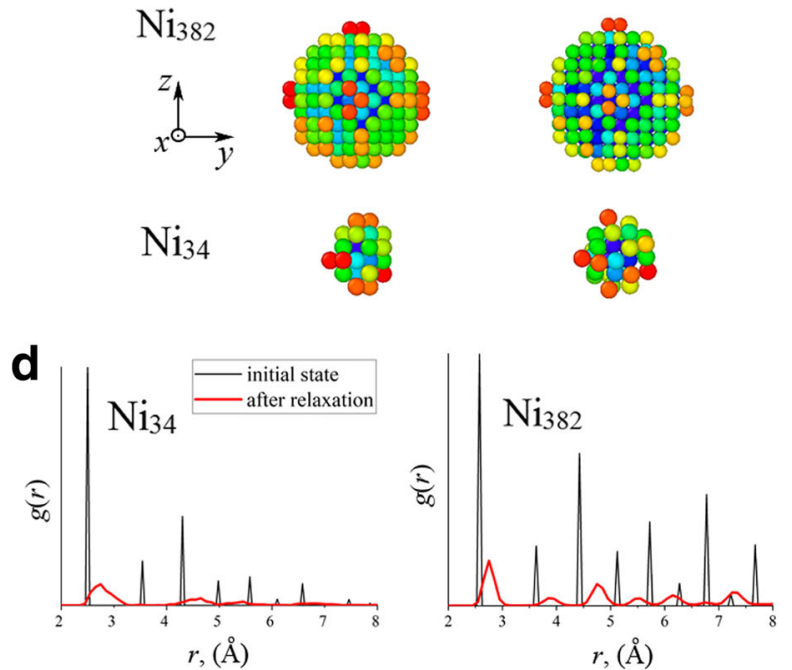

b
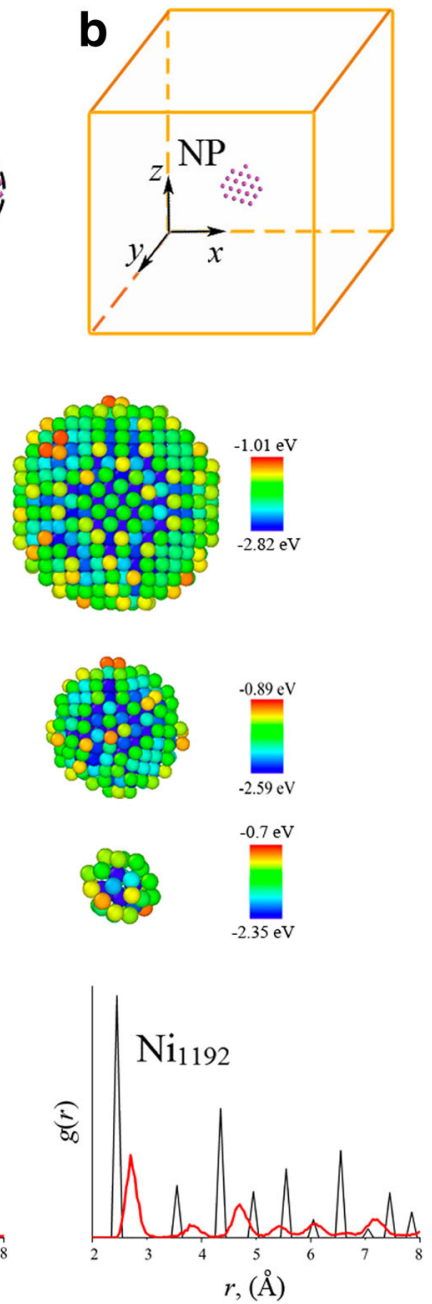

Fig. 1 a Schematic of the cutting of the NP from bulk Ni crystal. b Simulation cell. c Potential energy of atoms during relaxation. $\mathbf{d}$ Radial distribution function before and after relaxation

the biggest NP: covering of NP by graphene is expected in this case.

Molecular dynamics simulations are conducted using LAMMPS software environment. A Nose/Hoover thermostat is applied to maintain the temperature of $300 \mathrm{~K}$. The equations of motion were integrated using the Verlet algorithm with a time step of $10^{-15} \mathrm{~s}$.

\section{Results and discussion}

Melting of the nanoparticle

One of the aims of the present work is to check if the Morse potential suitable for such systems. At first, potential parameters for $\mathrm{Ni}$ are used to determine the phase transition of the cluster (or NP). It is well known that the melting temperature of small metal nanoparticles is lower than that of bulk materials, which occurs due to a substantial increase in the relative number of weakly bound atoms on the cluster surface. This temperature is point of phase transition at which the cluster transforms from a near-rigid, solid-like, structure to a nonrigid, liquid-like, structure but remains bound for thousands of vibrational periods. Disorder appearing first at the surface of the cluster as a result of the fact that the average coordination number of the surface atoms is less than that of the bulk atoms for a cluster of certain size. For example, melting of the isolated $\mathrm{Ni}_{309}$ nanoparticle simulated with the FinnisSinclair potential takes place in a broad temperature range between $900 \mathrm{~K}$ and $1400 \mathrm{~K}$ (Verkhovtsev et al. 2014).

In the present work, melting temperature of $\mathrm{Ni}_{34}$ is found to be $\sim 1360 \mathrm{~K}$. To monitor thermal behaviors of the NP, some physical quantities such as average potential energy per atom, radial atomic distribution, average interatomic distance as a function of temperature can be calculated. The roles of the surface and core atoms in the melting and fragmentation process of the cluster are also investigated by considering the surface and the bulk atoms 


\section{a}
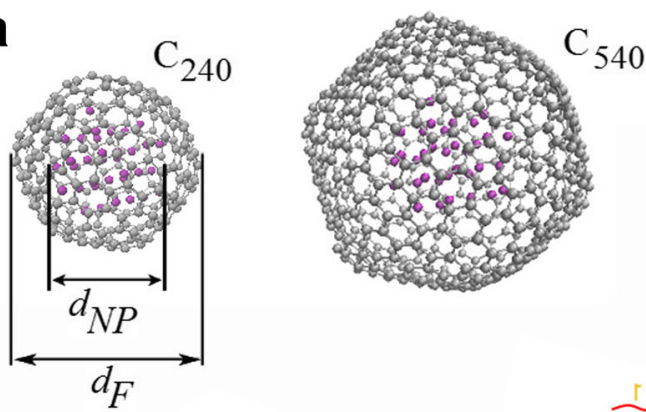

b
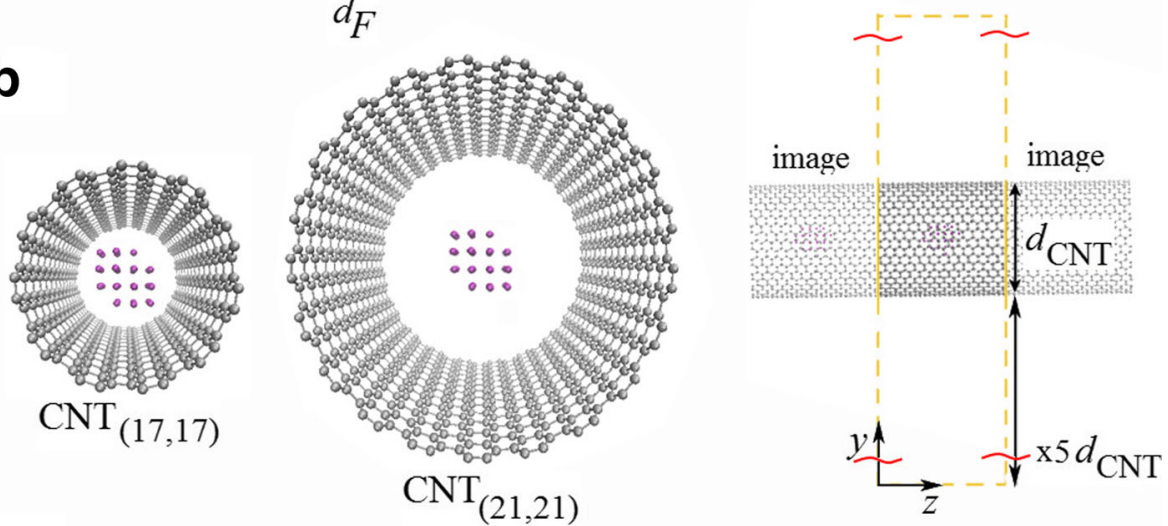

C
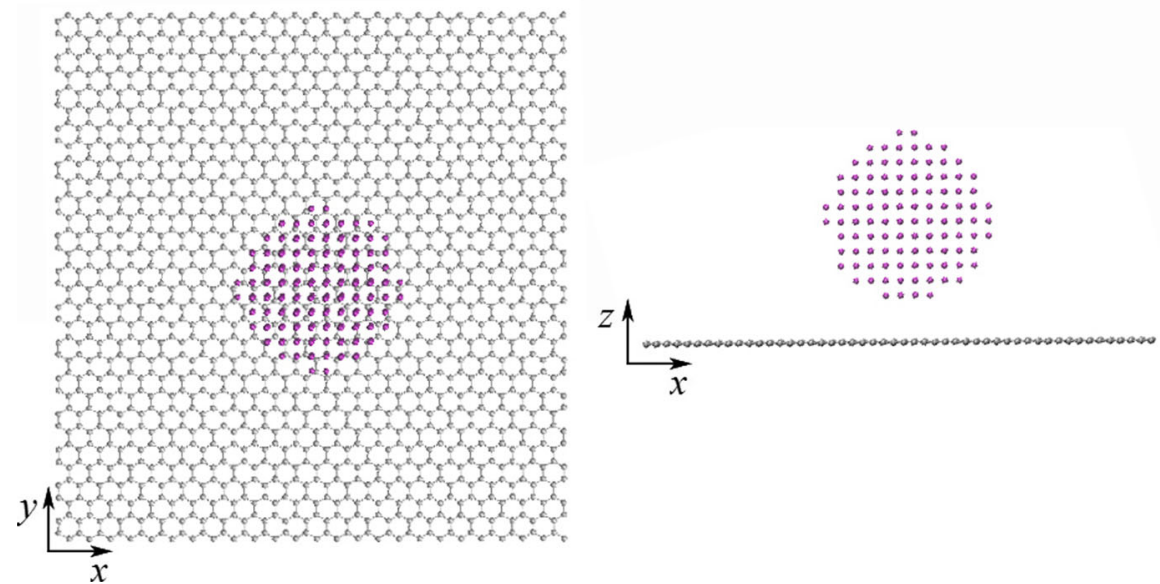

Fig. 2 Initial structures. a Ni nanoparticle inside the fullerenes $C_{240}$ and $C_{540}$. b Ni nanoparticle inside CNTs $(11,11),(21,21)$ and corresponding simulation cell. c Ni nanoparticle on graphene plane

of the NP. Here, the analysis is shown only for $\mathrm{Ni}_{34}$ as an example, but NP of other sizes are also studied in the same way.

In Fig. 3c, the caloric curve which is a plot of the average potential energy per atom versus temperature is shown for $\mathrm{Ni}_{34}$. Energetic state of the NP at different temperatures is also presented where each atom is colored in accordance with its potential energy. The average potential energy increases monotonically with temperature in the range $T \leq 1150 \mathrm{~K}$. Then, change in the average potential energy indicates that NP undergoes a phase change. As it can be seen, at $T=1000 \mathrm{~K}$, the nanoparticle is in the so-called pre-melted state, when the surface melting of the NP takes place, while the cluster core remains in the solid phase and resembles its crystalline structure. The average potential energy rises up from $1150 \mathrm{~K}$ to $1400 \mathrm{~K}$, which can be considered as the phase transition region where NP transforms from a near-rigid, solid-like structure to a nonrigid, liquid-like state, but remains bound for thousands of vibrational periods. For temperatures bigger than 1400 $\mathrm{K}$, increase of the potential energy of atoms take place and deattachment of the atoms from NP surface can be seen.

The plot of the average interatomic distance as a function of temperature (see Fig. 3b) is another indicator of 

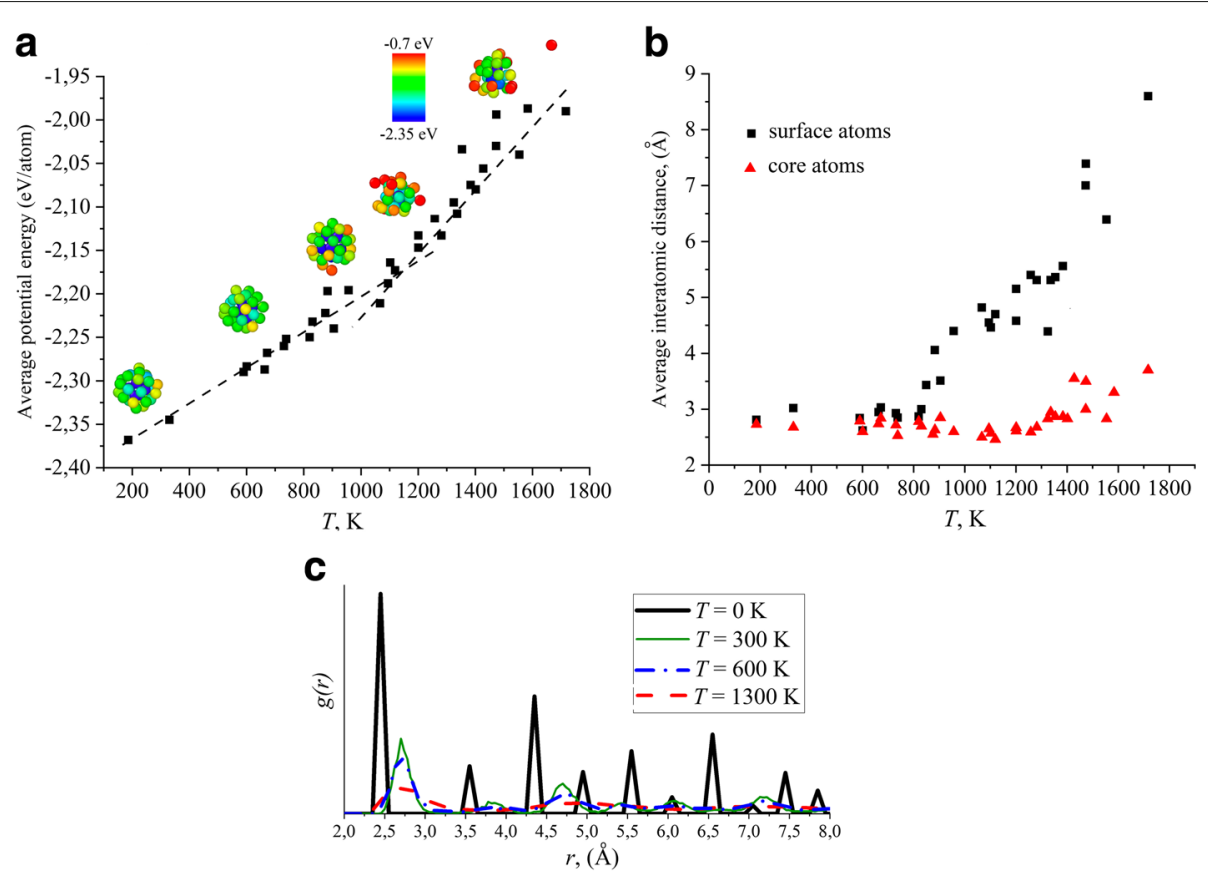

Fig. 3 a Average potential energy per atom versus temperature for $\mathrm{Ni}_{34}$. Atoms of the NP are colored in accordance with its potential energy. $\mathbf{b}$ The average interatomic distance as a function of temperature for $\mathrm{Ni}_{34}$ for surface atoms (black squares) and core atoms (red triangles). c Radial distribution function for temperatures 0 K, $300 \mathrm{~K}, 600 \mathrm{~K}$ and $1300 \mathrm{~K}$ for Ni $i_{382}$

thermal transition of $\mathrm{NP} \mathrm{Ni}_{34}$. Again, surface and core atoms of the NP behave differently and can be considered as the indicators of pre-melted and molten state. For surface atoms (shown by black squares), changes in the average interatomic distance starts at $900 \mathrm{~K}$ and continue to increase with the temperature. For core atoms (shown by red triangles), average interatomic distance starts to change at about $1150 \mathrm{~K}$ and further increase with the temperature.

Figure 3c shows radial distribution function for different temperatures for $\mathrm{Ni}_{382} \mathrm{NP}$, that reflects the information about the structure of NP as used previously (Mazzone 1998; Lewis et al. 1997). For $\mathrm{Ni}_{34}$ radial distribution function cannot be used to find the melting temperature, which was discussed above (see Fig. 1). But for $\mathrm{Ni}_{382}$, radial distribution function is analysed as the additional approvemen of the molten state. It can be seen, that at low temperature, the cluster consists first and second nearestneighbor peaks, but initial relaxation changes the value of the interatomic distance of NP from $2.49 \AA$ to $2.7 \AA$. On the other hand, at high temperature, the peaks are shapeless at large radius while the peaks are remaining the form at short radius which is typical of the liquid phase.

For comparison, melting point for Ni nanoparticle of 6 $\AA$ is estimated to be $1173 \mathrm{~K}$ (Fukuhara et al. 2017). Melting temperature of bigger Ni nanoparticles (20 $\AA$ and $26 \AA$ ) close to the limit for bulk Ni crystal ( $\sim 2000 \mathrm{~K})$. Very close critical temperatures was confirmed by realistic atomic potential function for Ni (Foiles et al. 1986). Since it is found, that nanoparticles with diameters bigger than $20 \AA$ have the melting temperature close to that of bulk $\mathrm{Ni}$ and can keep crystalline structure inside the NP, it is reasonable for further studies chose NPs of the same or bigger size to avoid the effect of chosen Morse parameters.

In Fig. 4a process of melting of Ni NP on graphene plane at $\mathrm{PBC}$ is shown for temperature range 1000-3000 K. While in Fig. 4b, the final structure of the melted $\mathrm{Ni}$ nanoparticle on graphene at free boundary conditions is presented. It can be seen, that melting starts between 2000 and $2200 \mathrm{~K}$ which can be additionally approved by radial distribution function for NP. In case of free boundary conditions, all $\mathrm{Ni}$ atoms interact with graphene plane, since graphene with free edges can bend and grab $\mathrm{Ni}$ atoms right after deattachment form the surface of Ni NP. In case of PBC, Ni atoms can separate from NP surface and move toward without any connection with graphene. Because of the finite size of the simulation cell and periodicity, some $\mathrm{Ni}$ atoms appear on the lower side of graphene plane, which does not mean that atoms move across graphene. For both cases, Ni atoms spreading on graphene surface and take the equilibrium position above the centre of carbon hexagon (region where it exactly seen is shown by red frame in Fig. 4b, the same is true for all the structure, but cannot be seen from this point of view). Connection of the $\mathrm{Ni}$ atoms to graphene surface results in bending of graphene, which was also mentioned in Katin et al. (2018). 


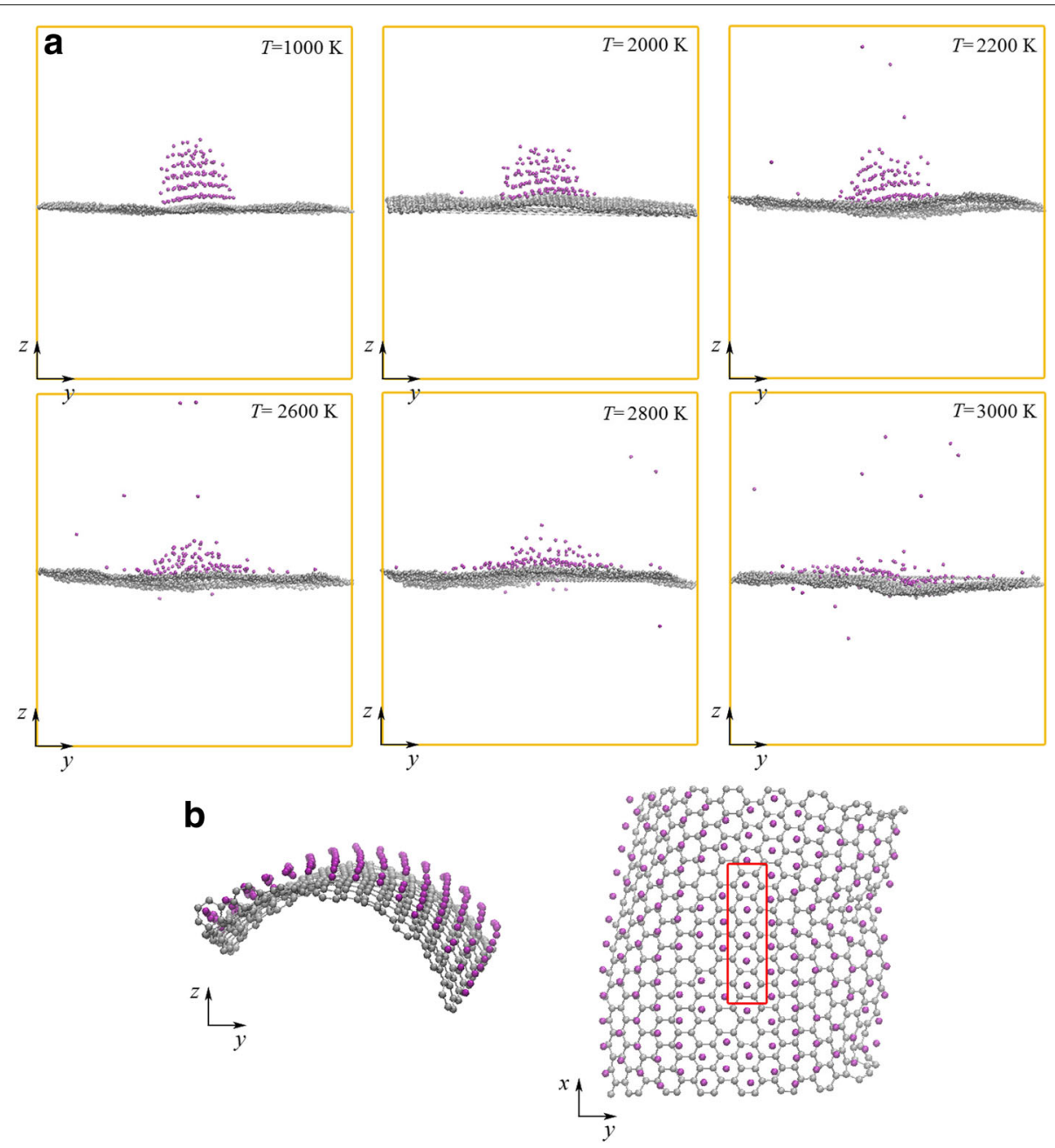

Fig. 4 a Process of melting of Ni NP on graphene plane at PBC. b Graphene plane covered by melted Ni NP at free boundary conditions in two projections

\section{Ni-carbon systems}

In Fig. 5, the snapshots of $\mathrm{Ni}_{34}$ nanoparticle inside the nanotubes $(11,11)$ and $(21,21)$ are shown. For both cases, $\mathrm{Ni}$ nanoparticle quickly move towards nanotube wall and fixes there. The size of $\mathrm{Ni}_{34}$ nanoparticle and nanotube $(11,11)$ is close which do not allow to characterize nanoparticle movement. Connection between the NP and nanotube wall lead to the corrugation of the nanotube. For the small nanotube, $\mathrm{Ni}$ atoms excite local movement of carbon atoms from the equilibrium positions. For bigger nanotube, overall change of the nanotube structure took place. The same is observed for fullerenes: NP move towards the wall and cause the change of spherical form of fullerene shell. After 5 ps, no other structural changes occur in the structure.

In Fig. 6, the snapshots of graphene-Ni nanoparticle system during simulation for (a) PBC and (b-d) free boundary conditions are shown for different simulation time.
Nanoparticles of three sizes are considered. Time $t=20 \mathrm{ps}$ is considered as final because at that time $\mathrm{Ni}_{1192} \mathrm{NP}$ covered by graphene from both sides an no further structural changes are achieved. The same is observed for two other NPs. Nevertheless, final structural configuration at $t=160$ ps is also shown for comparison. At initial state, the distance between graphene and bottom atoms of the NP is $\sim 4.8 \AA$. Ater relaxation, the distance between graphene and bottom atoms of the NP is $\sim 2.2 \AA$ which is close to the optimum interlayer distance between graphene and nickel layers equal to $2.1 \AA$ obtained by $a b$ - initio simulation (Hamada and Otani 2010).

In case of $\mathrm{PBC}, \mathrm{Ni}$ nanoparticle move towards graphene sheet (Fig. 6a). Slight corrugation of graphene is observed when the nanoparticle approaching the "infinite" graphene surface. When the edges of graphene flake are free and can interact with the other atoms, immediate corrugation of graphene took place with the simultaneous change of 

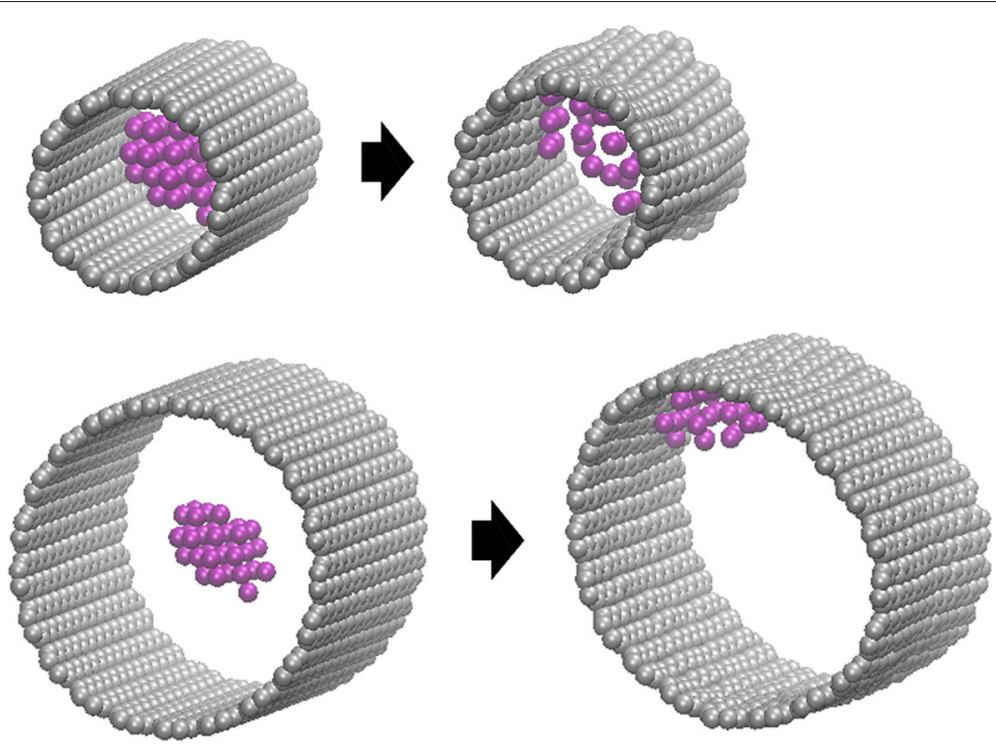

Fig. $5 \mathrm{Ni}$ nanoparticle inside carbon nanotubes $(11,11)$ and $(21,21)$ : initial and final stage at 5 ps

the perfectly spherical form of nanoparticle. The system tends to the full coverage of the Ni nanoparticle which correlates with the previous results (Shibuta and Maruyama 2007). However, for this initial set up full coverage cannot be achieved because of the process is strongly dependent on the size of NP. As it was shown in Bejagam et al. (2018), behaviour of the system, consisting of graphene and metal nanoparticle considerably depends on the NP size: small NP cannot be covered with graphene nanoribbon, while NP with $d_{N P}>40 \AA$ cause folding of graphene nanoribbon. The smallest NP act as a pin up for graphene plane. Small Ni cluster even spread a little on the graphene surface. NP with the average size can be covered much easier than $\mathrm{Ni}_{34} \mathrm{NP}$, but cannot be fully covered during this simulation time (160 ps). Graphene plane can be easily bended, but still, strong covalent bonding inside graphene plane makes the edges of structure (see Fig. $6 \mathrm{~d}$ at $t=160 \mathrm{ps}$ ) rigid. To fully cover the $\mathrm{Ni}_{1192} \mathrm{NP}$ additional external influence (for example, external pressure) is required.

For $\mathrm{Ni}_{34}$ and $\mathrm{Ni}_{382}$ NPs similar simulation was conducted with the help of ReaxFF potential for Ni-C (Bejagam et al. 2018). It is found that both potentials give qualitatively the same results. However, model with ReaxFF leads to a similar result for a significantly longer time than model with Morse potential. Therefore, ReaxFF is more suitable either for modeling small systems or processes occurring for short simulation time. Since the present model is developed to study carbon-nickel systems with the hundreds of thousands of atoms, Morse potential is much more suitable. Although, model with Morse potential give a bit stronger interaction between nanoparticle and carbon polymorphs.
For all graphene polymorphs and all the considered NPs the reconstruction of NP surface took place. NP, initially cut from face centered cubic crystal, cannot preserve highly symmetric order. The interconnection between nanoparticle and graphene leads to the small enlargement of the nanoparticle bottom, after which the nanoparticle is firmly fixing its place on graphene. Small nanocluster $\mathrm{Ni}_{34}$ became almost flat, while bigger NPs stay nearly spherical.

\section{Conclusions}

In the present study, the dynamics of $\mathrm{Ni}$ nanoparticle and the carbon nanopolymorphs is investigated by means of classical molecular dynamics simulations. In the performed simulations, the nickel-carbon interaction has been modeled by a pairwise Morse potential with the parameters found by ab-initio simulation in Katin et al. (2018).

It can be concluded, that:

1. Simple Morse potential can satisfactory reproduce the properties of Ni-carbon system. At the applied Morse parameters for $\mathrm{Ni}, \mathrm{Ni}_{34}$ is a cluster with the melting temperature $\sim 1360 \mathrm{~K}$, while melting temperature for $\mathrm{Ni}_{382}$ and $\mathrm{Ni}_{1192}$ close to the melting temperature of bulk $\mathrm{Ni}$ (about $2000 \mathrm{~K}$ ). These temperature values are in a good agreement with those from literature. Additional structural analysis have shown that the number of nickel atoms equal to 100 can be considered as critical transition between nanocluster and nanoparticle. Nanoclusters can easily lose their initial spherical shape, while NP undergo surface reconstruction but stay spherical. However, application of Morse parameters from Girifalco and Weizer (687) for NPs with the diameter smaller than $20 \AA$ should 

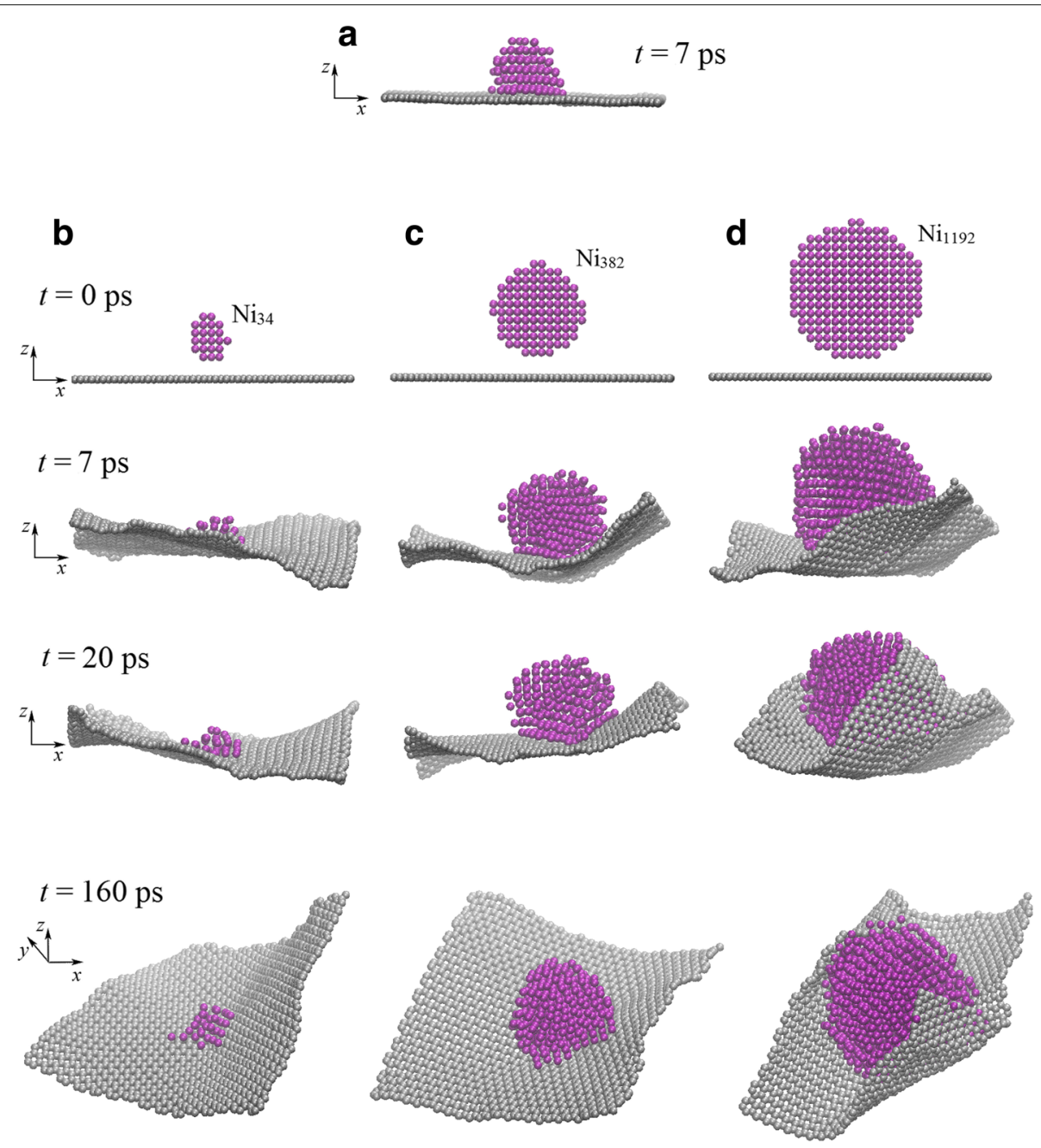

Fig. 6 Snapshots of graphene-Ni nanoparticle system during simulation:(a) PBC and (b-d) free boundary conditions. NP of three different sizes are considered. The time $t=160 \mathrm{ps}$ is the final stage of the simulation

be carefully checked. It is shown, that proposed model with the Morse potential can be successfully used for the numerical investigation of carbon-nickel structures.

2. Carbon nanopolymorphs are intensively contacting with the metal nanoparticle which allow fabricating insitu nano-composites with improved properties. Moreover, increasing of the temperature to melting level will not lead to the movement of $\mathrm{Ni}$ atoms from graphene surface or nanotube wall: $\mathrm{Ni}$ atoms are spreading on graphene/nanotube/fullerene shell. Nevertheless, vander-Waals forces connecting $\mathrm{Ni}$ atoms and the surface of carbon polymorph will be destroyed at high temperatures, which allow removing $\mathrm{Ni}$ atoms from carbon structure if required.

3. Ni NPs included to the pores of crumpled graphene will be easily covered by graphene flakes. This will lead to the formation of the so called in-situ graphene-nickel composite. It is expected that such complex structure will have improved mechanical properties $(\mathrm{Hu}$ et al. 2016; Jiang et al. 2018). Thus, the transition from carbon nanopolymorphs with nanoparticles to the complex three-dimensional systems is the further extension of the present work.

The graphene-metal composites will have considerably different physical properties. For example, the interaction between graphene and nickel can cause changes in the graphene electronic band structure which will inevitably affect the transport of electrons in the structure. Composite system will exhibit attractive features like high gravimetric density of hydrogen storage and low activation temperature for hydrogen release (Zhou et al. 2016); can be used for energy storage (Shi et al. 2016); and in practical applications in chemical and biological detection (Liu et al. 2014). Moreover, metal NPs can be used to activate, guide, and stabilize various carbon nanostructures (Bejagam et al. 2018). On the basement of the proposed 
model, Ni NPs inside crumpled graphene and in graphene scrolls will be studied further. As it can be seen, several important characteristics can affect the resulting properties: type and size of metal NP, boundary conditions for the simulation cell, temperature, Morse parameters for $\mathrm{Ni}$ nanoparticle, to name a few. The present work is the first step of a complex study of graphene-metal nanocomposites. Molecular dynamics simulation will allow to making a full analysis of the system parameters and define main features.

\section{Acknowledgements}

Not applicable.

\section{Funding}

Initial simulation setup was supported by the grant of the President of the Russian Federation for state support of young Russian scientists - doctors of sciences MD-1651.2018.2. Calculations were supported by the state assignment of IMSP RAS. The simulations were partly carried out on the supercomputer of RAS Supercomputer Center.

\section{Availability of data and materials}

The raw/processed data required to reproduce these findings cannot be shared at this time as the data also forms part of an ongoing study.

\section{Authors' contributions}

Initial simulation setup was conducted by SLR. The molecular dynamics study and drafting the manuscript were conducted by JAB and MRR. All authors read and approve the final manuscript.

\section{Competing interests}

None of the authors have any competing interests in the manuscript.

\section{Publisher's Note}

Springer Nature remains neutral with regard to jurisdictional claims in published maps and institutional affiliations.

Received: 8 July 2018 Accepted: 5 March 2019

Published online: 20 March 2019

\section{References}

Antoniammal P, Arivuoli D (2012) Size and Shape Dependence on Melting Temperature of Gallium Nitride Nanoparticles. J Nanomater 2012:11. http://dx.doi.org/10.1155/2012/415797

Babicheva RI, Baimova JA, Dmitriev SV, Pushin VG (2015) Two-dimensional model of the ordered alloy for the investigation of martensitic transformations. Lett Mater 5:359-63

Bai L, Srikanth N, Korznikova EA, Baimova JA, Dmitriev SV, Zhou K (2017) Wear and friction between smooth or rough diamond-like carbon films and diamond tips. Wear Elsevier BV 372-373:12-20

Baimova J, Murzaev R, Rudskoy A (2017) Discrete breathers in graphane in thermal equilibrium. Phys Lett A Elsevier BV 381:3049-53

Baimova JA, Liu B, Zhou K (2014) Folding and crumpling of graphene under biaxial compression. Lett Mater 4:96-9

Bejagam KK, Singh S, Deshmukh SA (2018) Nanoparticle activated and directed assembly of graphene into a nanoscroll. Carbon Elsevier BV 134:43-52

Brenner DW, Shenderova OA, Harrison JA, Stuart SJ, Ni B, Sinnott SB (2002) A second-generation reactive empirical bond order (REBO) potential energy expression for hydrocarbons. J Phys Condens Matter IOP Publ 14:783-802

Chetverikov A, Shepelev I, Korznikova E, Kistanov A, Dmitriev S, Velarde M (2017) Breathing subsonic crowdion in Morse lattices. Comput Condens Matter Elsevier BV 13:59-64

Dmitriev S, Kashchenko M, Baimova J, Babicheva R, Gunderov D, Pushin V (2017) Molecular dynamics simulation of the effect of dislocations on the martensitic transformations in a two-dimensional model. Lett Mater 7:442-6

Dmitriev SV, Khadeeva LZ, Pshenichnyuk Al, Medvedev NN (2010) Gap discrete breathers in two-component three-dimensional and two-dimensional crystals with Morse interatomic potentials. Phys Solid State Pleiades Publ Ltd 52:1499-505
Erkoc S, Gunes B, Gunes P (2000) Molecular-dynamics simulations of nickel clusters. Int J Mod Phys C 11(5):1013-24

Foiles SM, Baskes MI, Daw MS (1986) Embedded-atom-method functions for the fcc metals $\mathrm{Cu}, \mathrm{Ag}, \mathrm{Au}, \mathrm{Ni}, \mathrm{Pd}, \mathrm{Pt}$, and their alloys. Phys Rev B Am Phys Soc (APS) 33:7983-91

Fu Y, Cui WJ, Yan F, Zhao DY (2013) Synthesis of Graphene Load Nickel Nanoparticles Composites with Hydrothermal Process. Adv Mater Res Trans Tech Publ 760-762:793-6

Fukuhara S, Shimojo F, Shibuta Y (2017) Conformation and catalytic activity of nickel - carbon cluster for ethanol dissociation in carbon nanotube synthesis: Ab initio molecular dynamics simulation. Chem Phys Lett 679:164-71

Gaboardi M, Bliersbach A, Bertoni G, Aramini M, Vlahopoulou G, Pontiroli D, Mauron P, Magnani G, Salviati G, Züttel A, Riccò M (2014) Decoration of graphene with nickel nanoparticles: study of the interaction with hydrogen. J Mater Chem A R Soc Chem (RSC) 2:1039-46

Galashev AY, Katin KP, Maslov MM (2019) Morse parameters for the interaction of metals with graphene and silicene. Phys Lett A Elsevier BV 383:252-8

Geim AK, Novoselov KS (2007) The rise of graphene. Nat Mater Springer Nat 6:183-91

Gibson JS, Uddin J, Cundari TR, Bodiford NK, Wilson AK (2010) First-principle study of structure and stability of nickel carbides. J Phys Condens Matter IOP Publ 22:445503

Girifalco LA, Weizer VG (687) Application of the Morse Potential Function to Cubic Metals Physical Review. American Physical Society (APS) 114

Gunes B, Erkoc S (2000) Melting and fragmentation of Ni nanopartiles: molecular-dynamics simulation. Int J Mod Phys C 11(8):1567-80

Hamada I, Otani M (2010) Comparative van der Waals density-functional study of graphene on metal surfaces. Phys Rev B 82:153412

Hao Z, Cui R, Fan Y, Lin J (2019) Diffusion mechanism of tools and simulation in nanoscale cutting the $\mathrm{Ni}-\mathrm{Fe}-\mathrm{Cr}$ series of Nickel-based superalloy. Int J Mech Sci 150:625-36

Henz BJ, Hawa T, Zachariah MR (2009) Molecular Dynamics Simulation of the Kinetic Reaction of Ni and Al Nanoparticles. Mol Simul 35(10):804-811

Hu Z, Tong G, Lin D, Nian Q, Shao J, Hu Y, Saeib M, Jin S, Cheng GJ (2016) Laser sintered graphene nickel nanocomposites. J Mater Process Technol Elsevier BV 231:143-15

Huang H, Tang X, Chen F, Liu J, Sun X, Ji L (2018) Radiation tolerance of nickel-graphene nanocomposite with disordered graphene. J Nucl Mater Elsevier BV 510:1-9

lijima S, Ichihashi T (1993) Single-shell carbon nanotubes of 1-nm diameter. Nature Springer Nat 363:603-5

Ip S, Sridhar R, Toguri J, Stephenson T, Warner A (1998) Wettability of nickel coated graphite by aluminum. Mater Sci Eng A Elsevier BV 244:31-8

Ji L, Chen F, Huang H, Sun X, Yan Y, Tang X (2018) Preparation of nickel-graphene composites by jet electrodeposition and the influence of graphene oxide concentration on the morphologies and properties. Surf Coat Technol Elsevier BV 351:212-9

Jiang J, He X, Du J, Pang X, Yang H, Wei Z (2018) In-situ fabrication of graphene-nickel matrix composites. Mater Lett Elsevier BV 220:178-81

Joshi NP, Spearot DE, Bhat D (2010) Melting of Ni and Fe Nanoparticles: A Molecular Dynamics Study with Application to Carbon Nanotube Synthesis. J Nanosci Nanotechnol 10:5587-93

Katin KP, Prudkovskiy VS, Maslov MM (2018) Molecular dynamics simulation of nickel-coated graphene bending. Micro Nano Lett Inst Eng Technol (IET) 13:160-4

Krylova KA, Baimova YA, Dmitriev SV, Mulyukov RR (2016) Calculation of the structure of carbon clusters based on fullerene-like C24 and C48 molecules. Phys Solid State Pleiades Publ Ltd 58:394-401

Lewis $L$, Jensen P, Barrat $J L$ (1997) Melting, freezing, and coalescence of gold nanoclusters. Phys Rev B 56:2248

Lisovenko DS, Baimova YA, Rysaeva LK, Gorodtsov VA, Dmitriev SV (2017) Equilibrium structures of carbon diamond-like clusters and their elastic properties. Phys Solid State Pleiades Publ Ltd 59:820-8

Liu B, Reddy CD, Jiang J, Baimova JA, Dmitriev SV, Nazarov AA, Zhou K (2012) Morphology and in-plane thermal conductivity of hybrid graphene sheets. Appl Phys Lett AIP Publ 101:211909

Liu Y, Hu Y, Zhang J (2014) Few-Layer Graphene-Encapsulated Metal Nanoparticles for Surface-Enhanced Raman Spectroscopy. J Phys Chem C Am Chem Soc (ACS) 118:8993-8 
Los JH, Ghiringhelli LM, Meijer EJ, Fasolino A (2005) Improved long-range reactive bond-order potential for carbon. I. Construction. Phys Rev B $72: 214102$

Mahale NK, Ladhe RD, Attarde SB, Ingle ST (2014) Synthesis and the Structural Transformation of fcc to hcp in Ni-Graphene Nanocomposite by Simple Chemical Route via Sonication. J Nanoparticles Hindawi Limited 2014:1-7

Marks NA (2000) Generalizing the environment-dependent interaction potential for carbon. Phys Rev B 63:035401

Mazzone AM (1998) Molecular dynamics calculations of melting and fragmentation of Ar and Ag clusters. Phil Mag Lett 78:145

Murdick DA, Zhou XW, Wadley HNG, Nguyen-Manh D, Drautz R, Pettifor DG (2006) Analytic bond-order potential for the gallium arsenide system. Phys Rev B 73:045206

Murzaev R, Bachurin D, Korznikova E, Dmitriev S (2017) Localized vibrational modes in diamond. Phys Lett A Elsevier BV 381:1003-8

Neiva EG, Souza VH, Huang K, Pénicaud A, Zarbin AJ (2015) Graphene/nickel nanoparticles composites from graphenide solutions. J Colloid Interface Sci Elsevier BV 453:28-35

Neto AHC, Guinea F, Peres NMR, Novoselov KS, Geim AK (2009) The electronic properties of graphene. Rev Mod Phys Am Phys Soc (APS) 81:109-62

Neubauer E, Kitzmantel M, Hulman M, Angerer P (2010) Potential and challenges of metal-matrix-composites reinforced with carbon nanofibers and carbon nanotubes. Compos Sci Technol Elsevier BV 70:2228-36

Novoselov KS (2004) Electric Field Effect in Atomically Thin Carbon Films Science. Am Assoc Adv Sci (AAAS) 306:666-9

Pedrielli A, Taioli S, Garberoglio G, Pugno NM (2017) Designing graphene based nanofoams with nonlinear auxetic and anisotropic mechanical properties under tension or compression. Carbon Elsevier BV 111:796-806

Pettifor DG, Oleinik II (2000) Bounded Analytic Bond-Order Potentials for sigma and pi Bonds. Phys Rev Lett Bull Am Phys Soc (APS) 84:4124-7

Poletaev GM, Medvedeva ES, Zorya IV, Novoselova DV, Starostenkov MD (2017) Russ. Phys J 60:201

Ryu JH, Kim HY, Kim DH, Seo DH, Lee HM (2010) Immobilization of Au Nanoclusters Supported on Graphite: Molecular Dynamics Simulations. J Phys Chem C 114:2022-6

Savin AV, Korznikova EA, Lobzenko IP, Baimova YA, Dmitriev SV (2016) Symmetric scrolled packings of multilayered carbon nanoribbons. Phys Solid State Pleiades Publ Ltd 58:1278-84

Sharma A, Hickman J, Gazit N, Rabkin E, Mishin Y (2018) Nickel nanoparticles set a new record of strength. Nat Commun 9:4102

Shi Q, Cha Y, Song Y, Lee J-I, Zhu C, Li X, Song M-K, Du D, Lin Y (2016) 3D graphene-based hybrid materials: synthesis and applications in energy storage and conversion. Nanoscale R Soc Chem (RSC) 8:15414-47

Shibuta Y, Maruyama S (2007) Bond-order potential for transition metal carbide cluster for the growth simulation of a single-walled carbon nanotube. Comput Mater Sci Elsevier BV 39:842-8

Shiozawa H, Briones-Leon A, Domanov O, Zechner G, Sato Y, Suenaga K, Saito T, Eisterer M, Weschke E, Lang W, Peterlik H, Pichler T (2015) Nickel clusters embedded in carbon nanotubes as high performance magnets. Sci Rep Springer Nat 5:15033

Stillinger FH, Weber TA (1985) Computer simulation of local order in condensed phases of silicon. Phys Rev B Am Phys Soc (APS) 31:5262-71

Stuart SJ, Tutein AB, Harrison JA (2000) A reactive potential for hydrocarbons with intermolecular interactions. J Chem Phys 112:6472-86

Tersoff J (1986) New empirical model for the structural properties of silicon. Phys Rev Lett Am Phys Soc (APS) 56:632-5

van Duin ACT, Dasgupta S, Lorant F, Goddard WA (2001) ReaxFF:A Reactive Force Field for Hydrocarbons. J Phys Chem A Am Chem Soc (ACS) 105:9396-409

Verkhovtsev AV, Schramm S, Solov'yov AV (2014) Molecular dynamics study of the stability of a carbon nanotube atop a catalytic nanoparticle. Eur Phys J D 68:246

Yamaguchi Y, Maruyama S (1999) A molecular dynamics study on the formation of metallofullerene. Eur Phys J D Springer Nat 9:385-8

Yan Y, Zhou S, Liu S (2017) Atomistic simulation on nanomechanical response of indented graphene/nickel system. Comput Mater Sci Elsevier BV 130:16-20

Yang X, Wu S, Xu J, Cao B, To AC (2018) Spurious heat conduction behavior of finite-size graphene nanoribbon under extreme uniaxial strain caused by the AIREBO potential. Phys E Low-Dimensional Syst Nanostruct Elsevier BV 96:46-53
Yousefia M, Khoie MM (2015) Molecular dynamics simulation of Ni/Cu-Ni nanoparticles sintering under various crystallographic, thermodynamic and multi-nanoparticles conditions. Eur Phys J D 69:71

Zhan H, Zhang G, Tan VBC, Cheng Y, Bell JM, Zhang Y-W, Gu Y (2016) From brittle to ductile: a structure dependent ductility of diamond nanothread. Nanoscale R Soc Chem (RSC) 8:11177-84

Zhou C, Szpunar JA, Cui X (2016) Synthesis of Ni/Graphene Nanocomposite for Hydrogen Storage. ACS Appl Mater Interfaces Am Chem Soc (ACS) 8:15232-41

\section{Submit your manuscript to a SpringerOpen ${ }^{\circ}$ journal and benefit from:}

- Convenient online submission

- Rigorous peer review

- Open access: articles freely available online

- High visibility within the field

- Retaining the copyright to your article

Submit your next manuscript at $>$ springeropen.com 